\title{
Dianhydrogalactitol induces replication- dependent DNA damage in tumor cells preferentially resolved by homologous recombination
}

\author{
Beibei Zhai ${ }^{1,2}$, Anne Steinø ${ }^{3,4}$, Jeffrey Bacha ${ }^{3,4}$, Dennis Brown ${ }^{3,4}$ and Mads Daugaard ${ }^{1,2}$
}

\begin{abstract}
1,2:5,6-Dianhydrogalactitol (DAG) is a bifunctional DNA-targeting agent causing $\mathrm{N}^{7}$-guanine alkylation and inter-strand DNA crosslinks currently in clinical trial for treatment of glioblastoma. While preclinical studies and clinical trials have demonstrated antitumor activity of DAG in a variety of malignancies, understanding the molecular mechanisms underlying DAG-induced cytotoxicity is essential for proper clinical qualification. Using non-small cell lung cancer (NSCLC) as a model system, we show that DAG-induced cytotoxicity materializes when cells enter $S$ phase with unrepaired $N^{7}$-guanine DNA crosslinks. In S phase, DAG-mediated DNA crosslink lesions translated into replicationdependent DNA double-strand breaks (DSBs) that subsequently triggered irreversible cell cycle arrest and loss of viability. DAG-treated NSCLC cells attempt to repair the DSBs by homologous recombination (HR) and inhibition of the HR repair pathway sensitized NSCLC cells to DAG-induced DNA damage. Accordingly, our work describes a molecular mechanism behind $N^{7}$-guanine crosslink-induced cytotoxicity in cancer cells and provides a rationale for using DAG analogs to treat HR-deficient tumors.
\end{abstract}

\section{Introduction}

Historical data from preclinical studies and clinical trials support anti-neoplastic effects of 1,2:5,6-dianhydrogalactitol (DAG) analogs in a variety of cancer types, including leukemia, brain, cervical, ovarian, and lung cancers $^{1-6}$. In China, DAG is approved for the treatment of lung cancer ${ }^{7}$. Worldwide, lung cancer is the leading cause of cancer-related deaths. The 5-year relative survival rate for lung cancer is $15 \%$ for men and $21 \%$ for women. There are two major types of lung cancer, nonsmall cell lung cancer (NSCLC) and small cell lung cancer (SCLC). NSCLC accounts for $80-85 \%$ of all lung cancer

Correspondence: Mads Daugaard (mads.daugaard@ubc.ca)

${ }^{1}$ Vancouver Prostate Centre, Vancouver, BC V6H 3Z6, Canada

${ }^{2}$ Department of Urologic Sciences, University of British Columbia, Vancouver, BC V5Z 1M9, Canada

Full list of author information is available at the end of the article.

Edited by I. Amelio and approximately 57\% of newly diagnosed NSCLC patients present with stage IV metastatic disease. The median overall survival for patients with stage IV NSCLC is 4 months, and the 5 -year survival rate is only $4 \%^{8-10}$. Brain metastases occur frequently in NSCLC patients, contributing to the poor prognosis of this disease ${ }^{11}$. Currently, the mainstay treatments of primary and metastatic NSCLC include surgery, radiation therapy, chemotherapy, and targeted therapies with monoclonal antibodies or tyrosine kinase inhibitors (TKIs) in patients exhibiting epidermal growth factor receptor mutations ${ }^{12-15}$. However, the outcome of NSCLC patients remains poor mainly due to acquired platinum-based chemotherapy and TKI treatment resistance ${ }^{16}$.

DAG is a small water-soluble molecule that readily crosses the blood-brain barrier (BBB) and accumulates in primary and secondary brain tumors ${ }^{4,17}$. Perhaps for that reason, DAG displays strong activity in animal models of

\section{(c) The Author(s) 2018}

(c) Open Access This article is licensed under a Creative Commons Attribution 4.0 International License, which permits use, sharing, adaptation, distribution and reproduction cc) in any medium or format, as long as you give appropriate credit to the original author(s) and the source, provide a link to the Creative Commons license, and indicate if changes were made. The images or other third party material in this article are included in the article's Creative Commons license, unless indicated otherwise in a credit line to the material. If material is not included in the article's Creative Commons license and your intended use is not permitted by statutory regulation or exceeds the permitted use, you will need to obtain permission directly from the copyright holder. To view a copy of this license, visit http://creativecommons.org/licenses/by/4.0/. 
metastatic NSCLC, including TKI-resistant NSCLC ${ }^{18}$. Informed by preclinical studies, DAG may have a therapeutic advantage as compared to other DNA crosslinking agents ${ }^{3,5}$.

Due to its ability to cross the BBB, DAG is currently being tested in patients with temozolomide (TMZ) refractory glioblastoma multiforme $(\mathrm{GBM})^{19,20}$. A recently completed phase I/II clinical trial in adult refractory GBM patients established a well-tolerated dosing regimen of DAG and confirmed myelosuppression as the dose-limiting toxicity with complete reversion upon treatment termination ${ }^{21}$. However, despite encouraging preclinical and clinical data in NSCLC and GBM, timely advancement of DAG analogs toward the clinical arena is hampered by inadequate understanding of the molecular mechanisms responsible for DAGmediated cytotoxicity in cancer cells. We therefore used NSCLC as a model system to investigate the mechanisms of cytotoxicity imposed by the clinical-grade DAG analog VAL- $083^{22}$.

\section{Results}

Loss of lung cancer cell viability after DAG treatment

To investigate the effects of DAG on lung cancer cells, we evaluated the cytotoxic activities of VAL-083 in a panel of NSCLC cell lines. Treatment of A549, H2122, and $\mathrm{H} 1792$ cells with $10 \mu \mathrm{M}$ VAL-083 for $72 \mathrm{~h}$ resulted in dramatic morphological changes such as swelling and cell detachment (Fig. 1a). To further characterize the effect of DAG on tumor cells, we treated H1792, H2122, $\mathrm{H} 23$, and A549 NSCLC cell lines with different concentrations of VAL-083 for $72 \mathrm{~h}$ and subsequently determined viability of each cell line. The analysis showed a concentration-dependent loss of viability in all VAL-083treated cell lines with half-maximal inhibitory concentration $\left(\mathrm{IC}_{50}\right)$ values in the low $\mu \mathrm{M}$ concentration range (Fig. 1b). In summary, these data demonstrate cytotoxic effects of DAG on NSCLC cells.

\section{DAG induces persistent DNA damage in lung cancer cells}

Many chemotherapeutic drugs work by inducing different types of DNA damage in rapid-dividing cancer cells. DAG has been reported to have bifunctional DNAtargeting activity leading to the formation of $\mathrm{N}^{7}$-monoalkylguanine and inter-strand DNA crosslinks ${ }^{22}$. To investigate the effects of DAG on DNA integrity, we examined VAL-083-treated NSCLC cells for phosphorylated histone variant $\mathrm{H} 2 \mathrm{AX}$ ( $\mathrm{\gamma} \mathrm{H} 2 \mathrm{AX}$ ), an extensively used surrogate marker of DNA double-strand breaks (DSBs) $^{23,24}$. Biochemical assessment of A549, H1792, and H2122 cells treated with VAL-083 for $24 \mathrm{~h}$ followed by removal of the drug showed strong $\mathrm{yH} 2 \mathrm{AX}$ expression that persisted for $72 \mathrm{~h}$ after drug removal (Fig. 2a). This was supported by an immunofluorescence (IF) imaging analysis showing sustained $\mathrm{\gamma H} 2 \mathrm{AX}$ foci formation in $90-100 \%$ of the cells (Fig. $2 \mathrm{~b}$ ) over a similar time frame (Fig. 2c). These data suggest that DAG induces DNA DSBs in NSCLC cells that cannot be repaired

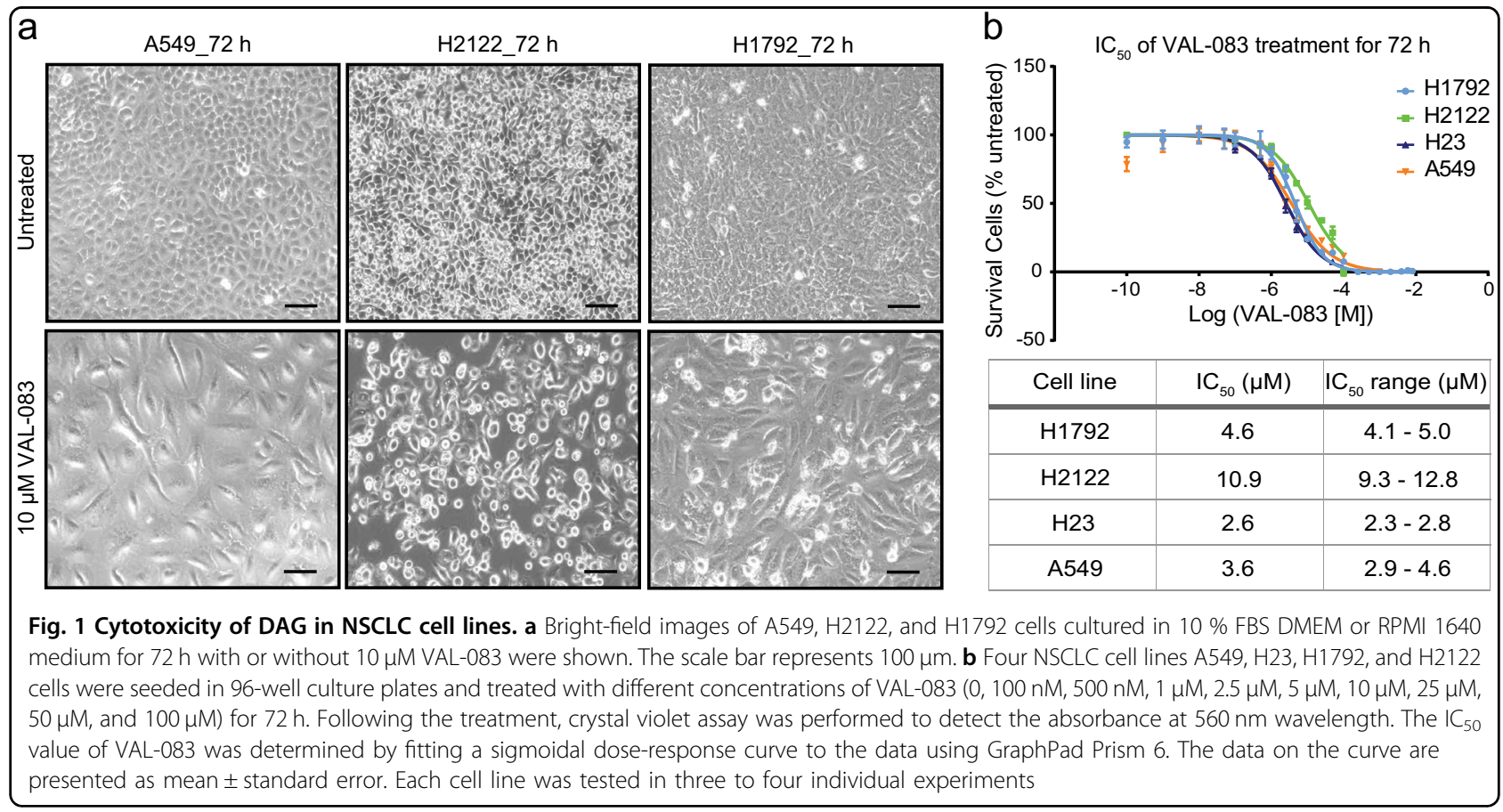




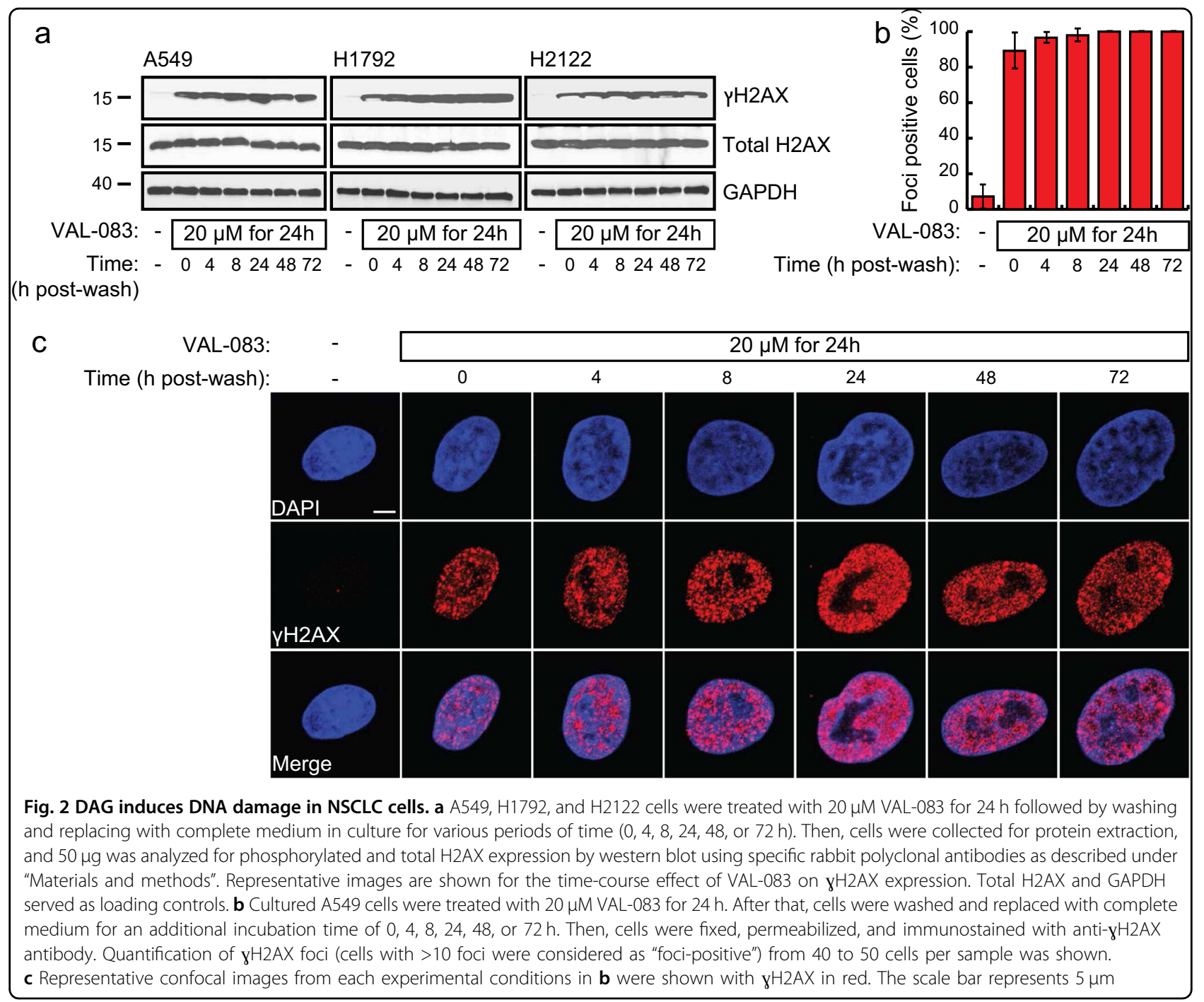

within a $72 \mathrm{~h}$ recovery period. In addition, VAL-083 induced strong $\mathrm{\gamma H} 2 \mathrm{AX}$ expression with at least $10 \mathrm{~h}$ incubation time (Supplementary Fig. S1a) and showed dose-dependent pattern (Supplementary Fig. S1b).

\section{Replication-dependent DNA damage of NSCLC cells upon DAG treatment}

We next investigated the effect of DAG on cell cycle progression using flow cytometric analysis of propidium iodide (PI)-stained NSCLC cells. VAL-083 treatment induced a strong dose-dependent $\mathrm{S} / \mathrm{G}_{2}$-phase cell cycle arrest in A549 (Fig. 3a) and H1792 (Fig. 3b) NSCLC cells. This result suggests that DAG-mediated cytotoxicity likely depends on replication. To further determine the role of replication for DAG-induced cytotoxicity, we synchronized the majority of A549 cells in $G_{0} / G_{1}$ phase by serum starvation for $24 \mathrm{~h}$ (Fig. 3c). Cells were then released from the cell cycle arrest by addition of serum with or without $5 \mu \mathrm{M}$ VAL-083 and followed by flow cytometry. While untreated cells rapidly assumed a normal cell cycle profile after serum addition, cells subjected to VAL-083 displayed a time-dependent $S / G_{2}$ phase arrest visual at and after $19 \mathrm{~h}$ in complete medium (Fig. 3c). Notably, this concentration of VAL-083 treatment did not increase the sub- $G_{1}$ population of cells (DNA content $<2 \mathrm{~N}$ reflects cellular debris and apoptotic cells) indicating that the time-dependent decrease in $G_{0} / G_{1}$ was not due to increased cell death (Fig. 3c). In parallel western blot analysis of cleaved caspase 3 expression confirmed the lack of apoptosis in VAL-083-treated cells (Supplementary Fig. S2).

Cyclins and cyclin-dependent kinases (CDKs) are key regulators of eukaryotic cell cycle progression. Activation of cyclin B-Cdc2 kinase complexes trigger M-phase entry, whereas cyclin A-Cdk2 complexes control the progression through $\mathrm{S}$ phase $\mathrm{e}^{25-27}$. Accordingly, cyclin expression is 


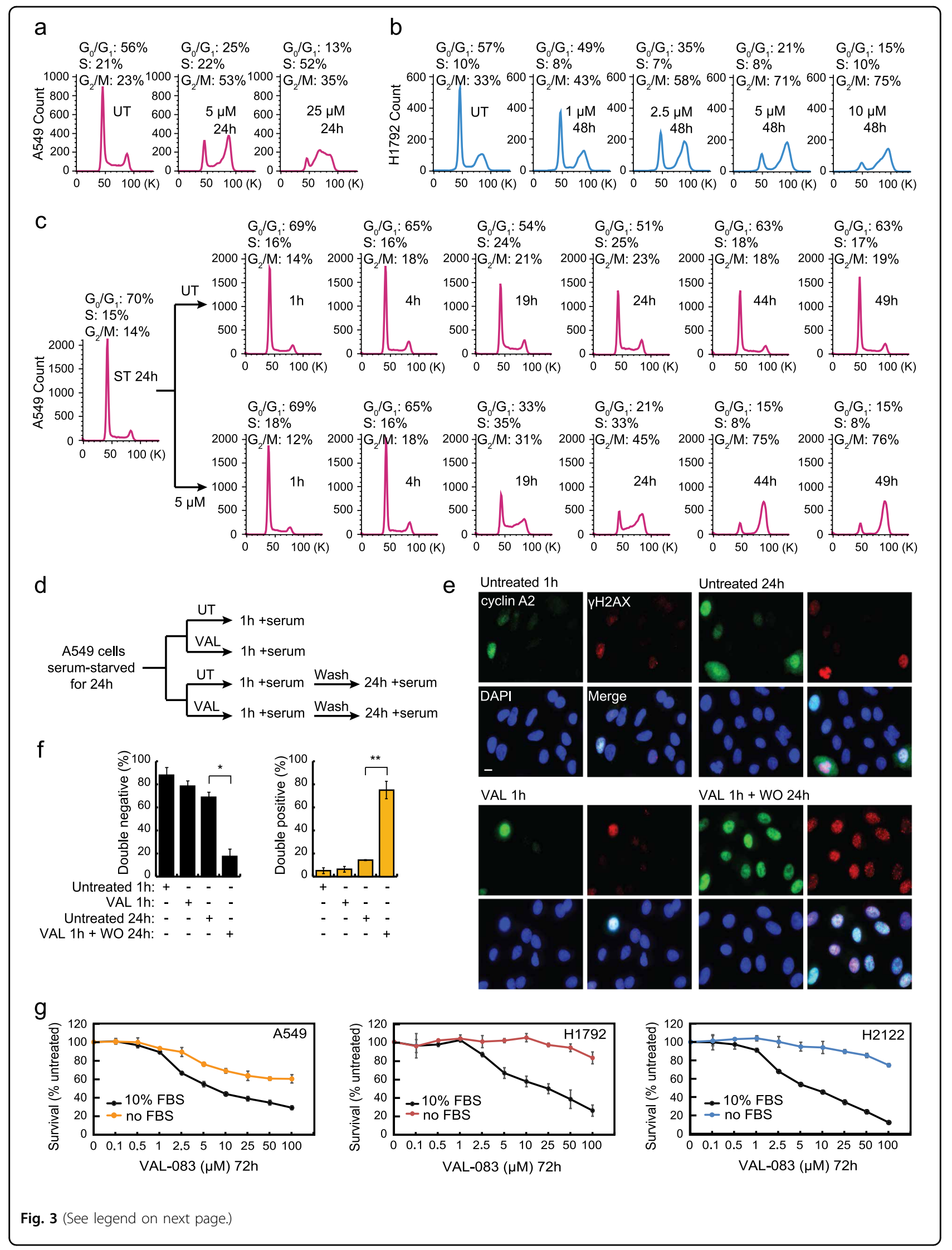


(see figure on previous page)

Fig. 3 DAG-induced DNA damage occurs in S phase of the cell cycle. a A549 cells were treated with 5 or $25 \mu \mathrm{M}$ VAL-083 for $24 \mathrm{~h}$. After that, cells were collected for cell cycle analysis using PI staining by flow cytometry. b After treatment with different concentrations of VAL-083 (1, 2.5, 5, or $10 \mu \mathrm{M})$ in $\mathrm{H} 1792$ cells for $48 \mathrm{~h}$, cell cycle analysis with PI staining was performed using flow cytometry. c A549 cells were synchronized by serum starvation (ST) for $24 \mathrm{~h}$ before treatment with or without $5 \mu \mathrm{M}$ VAL-083 for the indicated time periods $(1,4,19,24,44$, or $49 \mathrm{~h})$. Cells were then collected, fixed, and subsequently stained with PI for cell cycle distribution analysis by flow cytometry. For additional experimental details, see "Materials and methods". The representative flow cytometric plots from two individual experiments are shown. d Flow chart outlining the experimental conditions used in e. e A549 cells (synchronized by $24 \mathrm{~h}$ serum starvation) were treated with $50 \mu \mathrm{M}$ VAL-083 for $1 \mathrm{~h}$ and replaced with complete medium for another $24 \mathrm{~h}$ incubation. Then, cells were fixed, permeabilized, and immunostained with anti-cyclin A2 and anti-yH2AX antibodies. Representative IF images are shown with cyclin A2 in green and $\mathrm{yH} 2 \mathrm{AX}$ in red. The scale bar represents $10 \mu \mathrm{m}$. $\mathbf{f} \mathrm{In}$ all, $100-120$ cells were examined from each treatment condition as in $\mathbf{e}$. The percentages of two categories (cyclin A2-/yH2AX- and cyclin A2+/yH2AX+) of A549 cells are shown with corresponding statistical analysis $\left(^{*} p \leq 0.05\right.$; ${ }^{* *} p \leq 0.01$; Student's $t$ test). g A549 cells were seeded with either serum-deprived or complete medium in 96-well culture plates. After $24 \mathrm{~h}$ incubation, cells were treated with 0, $100 \mathrm{nM}, 500 \mathrm{nM}, 1 \mu \mathrm{M}, 2.5 \mu \mathrm{M}, 5 \mu \mathrm{M}, 10 \mu \mathrm{M}, 25 \mu \mathrm{M}, 50 \mu \mathrm{M}$, or $100 \mu \mathrm{M}$ VAL-083 for $72 \mathrm{~h}$. Following the treatment, the percentage of survival cells compared to the untreated condition was determined by crystal violet assay. The data are presented as mean \pm standard error. $\mathrm{H} 1792$ and $\mathrm{H} 2122$ cells were also tested in the same experimental condition

tightly regulated throughout the cell cycle where cyclin A is expressed exclusively in $S$ phase plateauing in $G_{2}$ phase $^{28}$. Using cyclin $\mathrm{A}$ as a marker of $\mathrm{S}$ phase entry, we performed IF staining for cyclin A2 and $\mathrm{\gamma} \mathrm{H} 2 \mathrm{AX}$ on A549 cells with or without VAL-083 pulse treatment $(50 \mu \mathrm{M}$ VAL-083 for $1 \mathrm{~h}$ ) (Fig. 3d). The analysis showed that synchronized A549 cells displayed a dramatic accumulation of cyclin A2 (green) and $\mathrm{yH} 2 \mathrm{AX}$ (red) expression after pulse treatment with VAL-083 followed by $24 \mathrm{~h}$ in complete medium (Fig. 3e). Importantly, the population of cells double-positive for cyclin $\mathrm{A} 2+/ \mathrm{\gamma} \mathrm{H} 2 \mathrm{AX}+$ were significantly increased after VAL-083 pulse treatment for $1 \mathrm{~h}$ followed by washout for $24 \mathrm{~h}$ (VAL $1 \mathrm{~h}+$ WO $24 \mathrm{~h}$ ) while double-negative cyclin $\mathrm{A} 2-/ \mathrm{yH} 2 \mathrm{AX}-$ cells decreased (Fig. 3f). This indicates that VAL-083-induced DNA ${ }^{7}$-guanine crosslinks translate into DSBs in $S / G_{2}$ phase of the cell cycle. To further validate the observed replication-dependent cytotoxicity of VAL-083 in NSCLC cell lines, we compared survival rates of cells cultured in complete medium with cells cultured in serum-deprived medium, challenged with increasing concentrations of VAL-083. Indeed, A549, H1792, and H2122 cells prohibited from entering $\mathrm{S}$ phase due to serum deprivation all showed resistance to VAL-083-induced cytotoxicity (Fig. 3g). Combined, these data suggest that DAG-induced cytotoxicity materializes as NSCLC cells progress into $\mathrm{S}$ phase of the cell cycle with unrepaired $\mathrm{N}^{7}$-guanine DNA crosslink lesions.

\section{DAG-induced DNA damage is preferentially repaired by homologous recombination}

As DAG induces DNA DSBs in S phase, cytotoxicity in cancer cells likely depends at least partially on their ability to repair DNA in $S / G_{2}$ phase of the cell cycle. DNA DSBs can be repaired by either non-homologous end joining (NHEJ) or homologous recombination (HR). While NHEJ can occur throughout the cell cycle, $\mathrm{HR}$ is restricted to the $\mathrm{S}$ and $\mathrm{G}_{2}$ phases where sister chromatids are available as templates for sequence homology-guided repair ${ }^{29}$. Since DAG-induced DNA DSBs materialized specifically in $\mathrm{S} / \mathrm{G}_{2}$, we hypothesized that NSCLC cells would repair these lesions by HR. To test this hypothesis, we monitored DNA DSB sensors and effectors involved in the HR pathway in NSCLC cells after VAL-083 treatment. VAL083 pulse treatment followed by $20-48 \mathrm{~h}$ incubation in complete medium without VAL-083 triggered the activation of ataxia telangiectasia mutated (ATM) kinase in A549, H1792, and H2122 cells (Fig. 4a and Supplementary Fig. S3). This was associated with threonine 68 phosphorylation of $\mathrm{S}$ phase checkpoint kinase Chk2 and the presence of $\gamma \mathrm{H} 2 \mathrm{AX}$. Upon activation of HR repair, the DNA surrounding the DSBs is processed in an enzymatic step that depends on CtIP and hepatoma-derived growth factor family co-factors to create a single-stranded DNA (ssDNA) template for homology pairing ${ }^{30-33}$. The resected ssDNA is subsequently occupied by ssDNA-binding replication protein A (RPA32) that is phosphorylated on serine 33 by the ataxia telangiectasia and Rad3-related protein (ATR) kinase in response to ssDNA exposure ${ }^{34}$. This event triggers the final step in the HR pathway where homology repair is completed in a Rad51-dependent manner ${ }^{35}$. Indeed, VAL-083 treatment induced phosphorylation of RPA32 as well as the Chk1 kinase, a wellknown phospho-target of ATR (Fig. 4a). In aggregate, these data suggest that NSCLC cells repair DAG-induced DSBs by HR. To further substantiate this finding, we analyzed the recruitment of key HR repair proteins to yH2AX foci in NSCLC cells treated with VAL-083 by confocal microscopy. The analysis showed that HR repair proteins BRCA1, RPA32, and Rad51 ${ }^{35}$ co-localized with $\mathrm{\gamma H} 2 \mathrm{AX}$ foci induced by VAL-083 (Fig. $4 \mathrm{~b}$ ) and these colocalizations were statistically significant (Fig. 4c). Combined, these data demonstrate that NSCLC cells repair DAG-induced DSBs by HR repair.

Our data entertain a prediction that cancer cells deficient in HR repair would have increased sensitivity to 


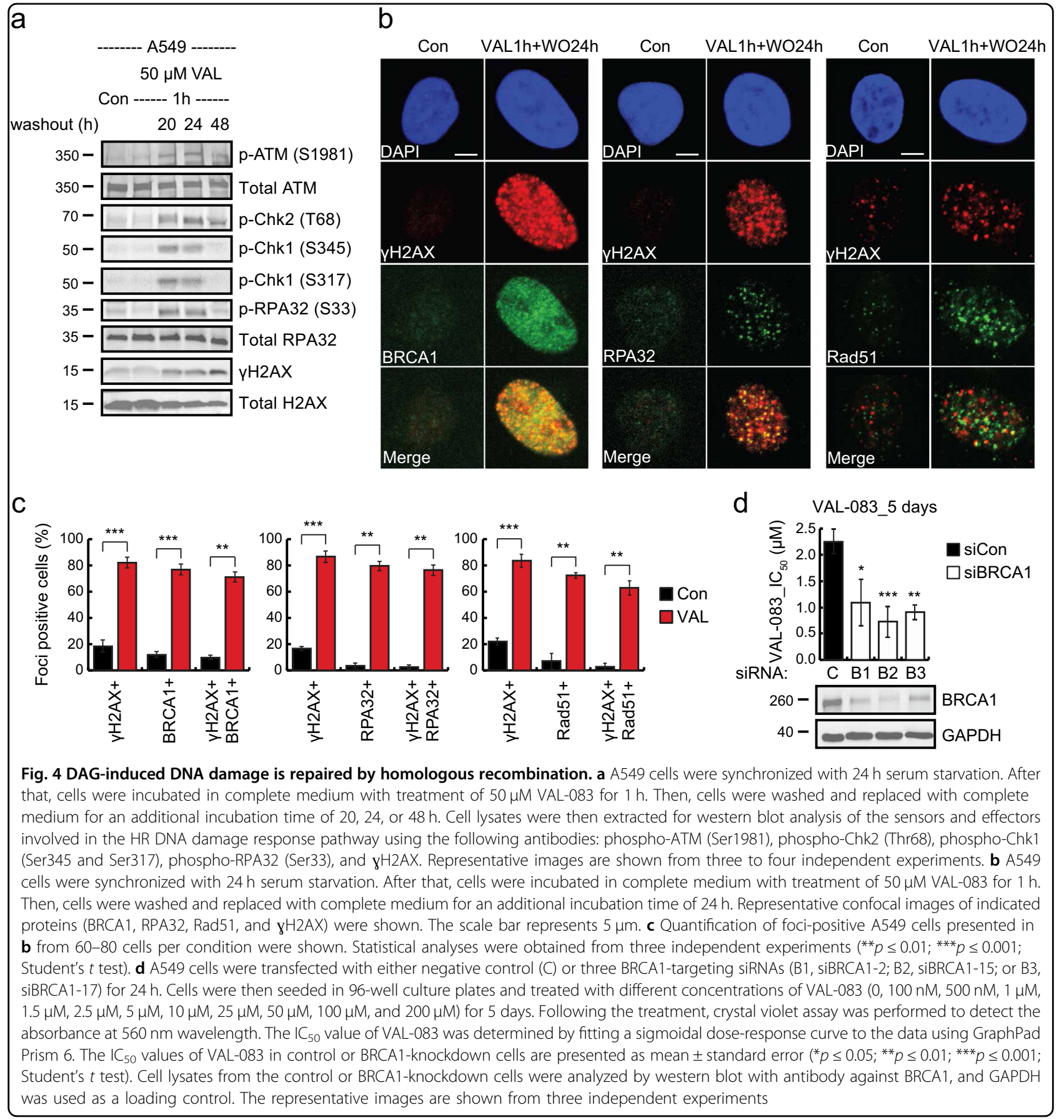

DAG. To confirm this prediction, we knocked down the essential HR repair protein BRCA1 ${ }^{36,37}$ in NSCLC cells using small interfering RNAs (siRNAs) before treatment with VAL-083. Indeed, knockdown of BRCA1 using three non-overlapping siRNAs significantly sensitized A549 cells to VAL-083 (Fig. 4d). These data confirm the prediction that cancer cells deficient in HR repair are unable to resolve DAG-induced DSBs.

\section{Discussion}

DNA crosslinking agents are widely used as chemotherapy in a variety of cancers ${ }^{38}$. While they all target DNA, there are small differences that distinguishes these agents from each other. For example, most agents have more than one function and it is often difficult to uncouple these functions in terms of mechanism of action. DAG is a bifunctional DNA-targeting agent causing 
$\mathrm{N}^{7}$-monoalkylguanine and inter-strand DNA crosslinks ${ }^{22}$, but it has also been reported to inhibit angiogenesis ${ }^{7}$. DAG has been shown to interact with DNA yielding 7-(1-deoxygalactit-1-yl)guanine, 7-(1-deoxyanhydrogalactit-1-yl)guanine, and 1,6-di(guanin-7-yl)-1,6-dideoxygalactitol, of which the last product indicates inter- or intra-strand crosslink formation $^{39}$. Up to this date, more than 40 NCIsponsored phase I and II clinical trials involving DAG has been conducted in the United States. Preclinical and clinical trial data suggest antitumor activity of DAG in several malignancies including lung cancer, brain tumors, leukemia, cervical cancer, and ovarian cancer ${ }^{1-6}$. Also, an openlabel post-market clinical trial in China investigates the activity of VAL-083 in relapsed or refractory NSCLC patients ${ }^{40}$.

TMZ is a DNA alkylating agent targeting $\mathrm{N}^{7}$ and $\mathrm{O}^{6}$ positions of guanine and is currently used as firstline treatment of $\mathrm{GBM}^{41}$. Interestingly, while $\mathrm{TMZ}$ and DAG have at least partially overlapping properties in terms of DNA interactions, mechanisms of cytotoxicity appear to be different. For example, GBM cells resistant to TMZ still show sensitivity to DAG ${ }^{42}$, indicating nonoverlapping functions between the two agents. Precise knowledge of the molecular mechanisms underlying tumor cell cytotoxicity is essential for optimal positioning of chemotherapeutic drugs in a clinical context. For that reason, we decided to dissect and describe the cytotoxic mechanisms of DAG using NSCLC as our model system. We found that VAL-083, a good manufacturing practice-produced clinical-grade DAG, is well-tolerated by cells as long as they are in $G_{1}$ phase of the cell cycle. Cells have several systems in place that can resolve DNA crosslinks and methylations such as nucleotide excision repair (intra-strand) and the Fanconi anemia system (inter-strand) ${ }^{38,43}$. Repair of DNA crosslinks in $G_{1}$ phase is supported by the $G_{1} / S$ phase checkpoint machinery that keeps cells in $G_{1}$ until repair has been completed $^{44}$. However, the $G_{1} / S$ phase checkpoint is often compromised in cancer cells and in this scenario, cells may enter $\mathrm{S}$ phase with unrepaired DNA lesions ${ }^{45}$. When the replication forks collide with the DNA lesions, replication is blocked exposing stretches of ssDNA that will subsequently be bound by RPA. The violent collision between the DNA lesion and the replication machinery will often result in DNA DSBs, which are catastrophic for the cells ${ }^{46}$. Indeed, we found the cytotoxic effect of DAG on NSCLC cells to develop in S phase indicating that these cells progress from $G_{1}$ to $S$ phase with unrepaired DNA lesions. In $S$ phase, DAG-treated cells display a profound $\mathrm{\gamma H} 2 \mathrm{AX}$ response indicative of DNA DSBs. We furthermore found that these DAG-induced DSBs activated the HR pathway and inhibition of HR dramatically sensitized NSCLC cells to DAG. As such, our data suggest that DAG-induced DNA

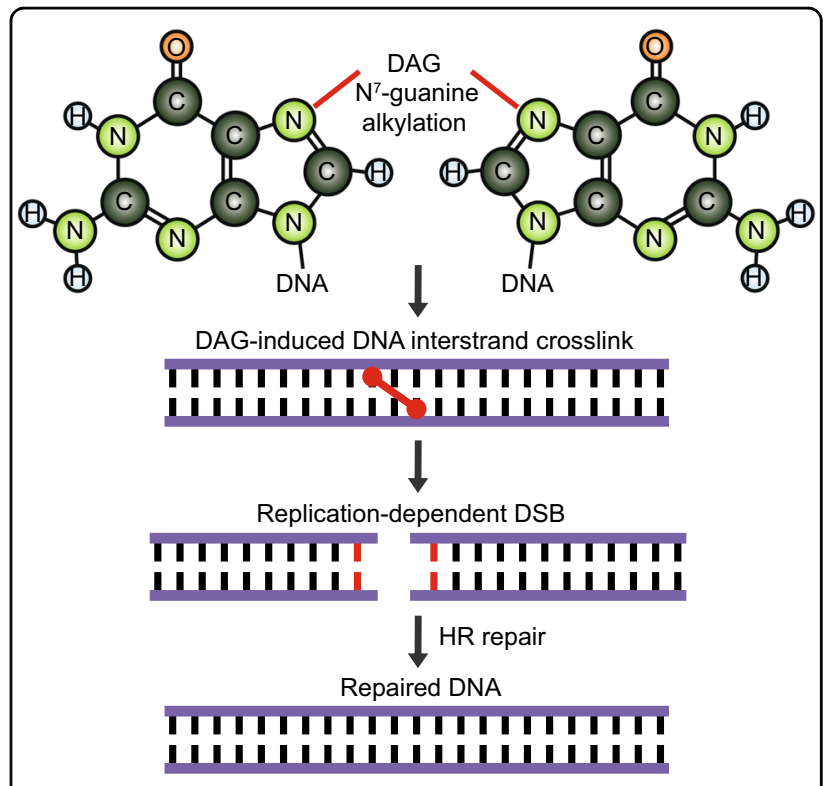

Fig. 5 Model of the mechanism of action of DAG in lung cancer cells. DAG treatment induces inter-strand DNA crosslinks through $\mathrm{N}^{7}$-guanine alkylation, leading to replication-dependent DNA doublestrand breaks (DSB). Lung cancer cells respond to the DAG-induced DNA DSB through activation of HR DNA repair

lesions translate into replication-dependent DNA DSBs in $S$ phase that are subsequently repaired by HR (Fig. 5). Importantly, our work provides a clinical rationale for positioning DAG-based chemotherapy in cancers deficient in HR repair.

\section{Materials and methods \\ Reagents and cell culture}

VAL-083 was obtained from DelMar Pharmaceuticals, Inc. (Vancouver, Canada and Menlo Park, CA, USA). PI solution $(1 \mathrm{mg} / \mathrm{ml})$ and glutaraldehyde solution (grade I, $50 \%$ in $\mathrm{H}_{2} \mathrm{O}$ ) were purchased from Sigma-Aldrich (Oakville, Canada). Sorenson's solution was prepared with $9 \mathrm{mg}$ trisodium citrate, $195 \mathrm{ml} 0.1 \mathrm{~N} \mathrm{HCl}, 500 \mathrm{ml} 90 \%$ ethanol, and $305 \mathrm{ml}$ distilled water. All cell lines were maintained at $37^{\circ} \mathrm{C}$ in $5 \% \mathrm{CO}_{2}$ atmosphere. A549 cells were cultured in Dulbecco's modified Eagle's medium supplemented with $10 \%$ fetal bovine serum. H2122, H1792, and H23 cells were cultured in RPMI 1640 with $10 \%$ fetal bovine serum.

\section{Crystal violet cell proliferation assay}

Following $72 \mathrm{~h}$ of different concentrations of VAL-083 treatment, cells were fixed in $1 \%$ glutaraldehyde solution for $5 \mathrm{~min}$. After rinsing with distilled water, cells were incubated with $0.1 \%$ crystal violet solution dye for $10 \mathrm{~min}$. Cells were then gently washed with distilled water and air-dried. The crystals on the plate were dissolved in Sorenson's solution before reading absorbance 
at $560 \mathrm{~nm}$ wavelength with a microplate reader. Survival cells were expressed as the percentage compared to untreated cells.

\section{Cell cycle analysis using PI staining}

Cell cycle distribution was evaluated based on DNA content using PI staining. Serum starvation (24 h)-synchronized cells were treated with $5 \mu \mathrm{M}$ VAL-083 for 1,4 , 19, 24, 44, and $49 \mathrm{~h}$. Cells were then trypsinized, washed in phosphate-buffered saline (PBS), and centrifuged at $1000 \mathrm{rpm}$ for $5 \mathrm{~min}$. Cell pellets were fixed in $70 \%$ ethanol at least overnight at $4{ }^{\circ} \mathrm{C}$. After washing with PBS, cells were incubated with $500 \mu \mathrm{l}$ PI solution in PBS containing $50 \mu \mathrm{g} / \mathrm{ml} \mathrm{PI}, 100 \mu \mathrm{g} / \mathrm{ml}$ RNase A, and 0.05\% Triton X-100 for $40 \mathrm{~min}$ at $37^{\circ} \mathrm{C}$ in the dark. Thereafter, cells were washed and resuspended in PBS. DNA content were analyzed by flow cytometry (FACS Canto II), and histograms and quantitative analyses of the proportions of cells in $G_{0} / G_{1}, S$ and $G_{2} / M$ phases were made using FlowJo software. Untreated cells were included as control.

\section{Western blotting}

Cells were lysed in EBC buffer $(50 \mathrm{mM}$ Tris- $\mathrm{HCl}$, $\mathrm{pH}$ 8.0, $120 \mathrm{mM} \mathrm{NaCl}, 1 \% \mathrm{NP}-40$, and $1 \mathrm{mM}$ EDTA) supplemented with phosphatase inhibitor and protease inhibitor (Roche, Mississauga, Canada). Cellular proteins were separated by SDS-polyacrylamide gel electrophoresis and transferred onto polyvinylidene fluoride membrane. After incubation with blocking buffer for $1 \mathrm{~h}$, the membrane was incubated with designated primary antibodies overnight at $4{ }^{\circ} \mathrm{C}$. Then, membrane was washed three times for $10 \mathrm{~min}$ with TBST and incubated with horseradish peroxidase-conjugated anti-mouse or anti-rabbit antibodies (Santa Cruz Biotechnology, Dallas, TX, USA) for 1-2 $\mathrm{h}$. Membrane was washed with TBST three times and developed with Pierce ECL substrate system (ThermoFisher Scientific, Burlington, Canada) according to the manufacturer's instruction. The following primary antibodies were used for immunoblotting: $\gamma \mathrm{H} 2 \mathrm{AX}$ (Cell Signaling Technology, Danvers, MA, USA, 2577); H2AX (Abcam, Toronto, Canada, ab11175); phospho-ATM (Ser1981) (Rockland Antibodies and Assays, Limerick, PA, USA, 200-301-400); ATM (Cell Signaling Technology, 2873); GAPDH (Cell Signaling Technology, 5174); phospho-RPA32 (Ser33) (Bethyl Laboratories, Montgomery, TX, USA, A300-246A); phospho-Chk1 (Ser345) (Cell Signaling Technology, 2348); phospho-Chk1 (Ser317) (Cell Signaling Technology, 12302); phosphoChk2 (Thr68) (Cell Signaling Technology, 2661); RPA32 (Abcam, ab2175); BRCA1 (Novus Biologicals, Oakville, Canada, NB 100-404); and cleaved caspase 3 (Cell Signaling Technology, 9661). Representative blotting images were shown from three to four independent experiments.

\section{IF and microscope}

Cells were grown on glass coverslips for at least $16 \mathrm{~h}$ before serum starvation for $24 \mathrm{~h}$. Synchronized cells were treated with $50 \mu \mathrm{M}$ VAL-083 for $1 \mathrm{~h}$ followed by washout and incubation with complete medium for another $24 \mathrm{~h}$. Subsequently, cells were washed once with PBS and fixed for $30 \mathrm{~min}$ with $4 \%$ paraformaldehyde in $\mathrm{PBS}$ at room temperature. For DNA damage foci detection, cells were pre-extracted with cytoskeletal buffer (25 mM HEPES, $\mathrm{pH}$ 7.4, $50 \mathrm{mM} \mathrm{NaCl}, 1 \mathrm{mM}$ EDTA, $3 \mathrm{mM} \mathrm{MgCl}_{2}, 300 \mathrm{mM}$ sucrose, and $0.5 \%$ Triton X-100) for $5 \mathrm{~min}$ at $4{ }^{\circ} \mathrm{C}$ before fixation with $4 \%$ paraformaldehyde solution. Fixed cells were washed three times with PBS and permeabilized for 20 min with $0.5 \%$ Triton X-100 in PBS. After washing with PBS for three times and blocking with $3 \%$ bovine serum albumin in PBS for $1 \mathrm{~h}$ at room temperature, cells were incubated overnight at $4{ }^{\circ} \mathrm{C}$ with corresponding primary antibodies diluted in blocking solution. The next day, cells were washed three times with PBS and incubated with appropriate fluorophore-labeled secondary antibodies for $1 \mathrm{~h}$ at room temperature. After washing with PBS for three times, the coverslips were mounted with Vectashield mounting medium (with 4',6-diamidino-2-phenylindole). Images were acquired using Zeiss AxioObserver microscope and confocal LSM-780 microscope. The LSM-ZEN software was used for analyzing the images. The following primary and secondary antibodies were used in IF staining: yH2AX (Cell Signaling Technology, 2577); cyclin A2 (Abcam, ab16726); yH2AX (EMD Millipore, Etobicoke, Canada, 05-636); BRCA1 (Abcam, ab16780); Rad51 (Santa Cruz Biotechnology, H8349); RPA32 (Abcam, ab2175); donkey anti-rabbit Alexa-Fluor 594 (ThermoFisher Scientific, A21207); donkey anti-rabbit Alexa-Fluor 488 (ThermoFisher Scientific, A21206); donkey anti-mouse Alexa-Fluor 594 (ThermoFisher Scientific, A21203); and donkey anti-mouse Alexa-Fluor 488 (ThermoFisher Scientific, A21202). Representative images were shown from three independent experiments.

\section{siRNA transfection}

A549 cells were transfected with either a control siRNA or siRNAs targeting BRCA1 using RNAiMAX transfection reagent (ThermoFisher Scientific) according to the manufacturer's instruction. After $24 \mathrm{~h}$ of transfection, A549 cells were seeded in 96-well culture plates and treated with different concentrations of VAL-083 for 5 days followed by crystal violet assay. In parallel cell lysates were collected for western blot verification of BRCA1 knockdown. The siRNAs used in this study were as follows: $\mathrm{C}$, siCon (negative control medium GC duplex, Invitrogen, 462001); B1, siBRCA1-2 (Qiagen, Toronto, Canada, SI00096313); B2, siBRCA1-15 (Qiagen, SI02664368); and B3, siBRCA1-17 (Qiagen, SI03103975). 


\section{Statistical analysis}

Where indicated, $p$-values were calculated using Student's $t$ test. Data were presented as mean \pm SD of three independent experiments.

\section{Acknowledgements}

We thank DelMar Pharmaceuticals for kindly providing us with VAL-083. This work was supported by National Research Council Industrial Research Assistance Program [IT06135]; Mitacs [F15-03171]; DelMar Pharmaceuticals, Inc. [F14-03316]; and the Vancouver Prostate Centre. Funding for open access charge: National Research Council Industrial Research Assistance Program.

\section{Author details}

${ }^{1}$ Vancouver Prostate Centre, Vancouver, BC V6H 3Z6, Canada. ${ }^{2}$ Department of Urologic Sciences, University of British Columbia, Vancouver, BC V5Z 1M9, Canada. ${ }^{3}$ DelMar Pharmaceuticals, Inc., Vancouver, BC V5Z 1K5, Canada. ${ }^{4}$ DelMar Pharmaceuticals, Inc., Menlo Park, CA 94025, USA

\section{Conflict of interest}

A.S., J.B., and D.B. are employees of DelMar Pharmaceuticals, Inc. The remaining authors declare no conflict of interest.

\section{Publisher's note}

Springer Nature remains neutral with regard to jurisdictional claims in published maps and institutional affiliations.

Supplementary Information accompanies this paper at (https://doi.org/ 10.1038/s41419-018-1069-9).

Received: 15 June 2018 Revised: 30 August 2018 Accepted: 12 September 2018

Published online: 03 October 2018

\section{References}

1. Nemeth, L. et al. Pharmacologic and antitumor effects of 1,2:5,6-dianhydrogalactitol (NSC-132313). Cancer Chemother. Rep. 56, 593-602 (1972).

2. Haas, C. D., Stephens, R. L., Hollister, M. \& Hoogstraten, B. Phase I evaluation of dianhydrogalactitol (NSC-132313). Cancer Treat. Rep. 60, 611-614 (1976).

3. Eagan, R. T. et al. Platinum-based polychemotherapy versus dianhydrogalactitol in advanced non-small cell lung cancer. Cancer Treat. Rep. 61, 1339-1345 (1977).

4. Eagan, R. T. et al. Dianhydrogalactitol and radiation therapy. Treatment of supratentorial glioma. JAMA 241, 2046-2050 (1979).

5. Eagan, R. T. et al. Phase II study of the combination of dianhydrogalactitol, doxorubicin, and cisplatin (DAP) in patients with advanced squamous cell lung cancer. Cancer Treat. Rep. 65, 517-519 (1981).

6. Haas, C. D., Baker, L. \& Thigpen, T. Phase II evaluation of dianhydrogalactitol in lung cancer: a Southwest Oncology Group Study. Cancer Treat. Rep. 65 115-117 (1981).

7. Jiang, X. et al. Dianhydrogalactitol, a potential multitarget agent, inhibits glioblastoma migration, invasion, and angiogenesis. Biomed. Pharmacother. 91, 1065-1074 (2017).

8. Cancer Facts \& Figures 2017. https://www.cancer.org/research/cancer-factsstatistics/all-cancer-facts-figures/cancer-facts-figures-2017.html (2017).

9. Cetin, K., Ettinger, D. S., Hei, Y. J. \& O'Malley, C. D. Survival by histologic subtype in stage IV nonsmall cell lung cancer based on data from the Surveillance, Epidemiology and End Results Program. Clin. Epidemiol. 3, 139-148 (2011).

10. Howlader, N. et al. SEER Cancer Statistics Review, 1975-2013. https://seer. cancer.gov/csr/1975_2013/ (2016).

11. Barnholtz-Sloan, J. S. et al. Incidence proportions of brain metastases in patients diagnosed (1973 to 2001) in the Metropolitan Detroit Cancer Surveillance System. J. Clin. Oncol. 22, 2865-2872 (2004).

12. Pfister, D. G. et al. American Society of Clinical Oncology treatment of unresectable non-small-cell lung cancer guideline: update 2003. J. Clin. Oncol. 22 330-353 (2004).
13. Pisters, K. M. et al. Cancer Care Ontario and American Society of Clinical Oncology adjuvant chemotherapy and adjuvant radiation therapy for stages I-IIIA resectable non small-cell lung cancer guideline. J. Clin. Oncol. $\mathbf{2 5}$ 5506-5518 (2007)

14. Azzoli, C. G. et al. American Society of Clinical Oncology Clinical Practice Guideline update on chemotherapy for stage IV non-small-cell lung cancer. J. Clin. Oncol. 27, 6251-6266 (2009).

15. Minari, R., Bordi, P. \& Tiseo, M. Third-generation epidermal growth factor receptor-tyrosine kinase inhibitors in T790M-positive non-small cell lung cancer: review on emerged mechanisms of resistance. Transl. Lung Cancer Res. 5, 695-708 (2016).

16. Chang, A. Chemotherapy, chemoresistance and the changing treatment landscape for NSCLC. Lung Cancer 71, 3-10 (2011).

17. Eckhardt, S. et al. Uptake of labeled dianhydrogalactitol into human gliomas and nervous tissue. Cancer Treat. Rep. 61, 841-847 (1977).

18. Steino, A. et al. In vivo efficacy of VAL-083 in the treatment of non-small cell lung cancer. Cancer Res. 74, 824 (2014). abstract.

19. Fouse, S. D. et al. Dianhydrogalactitol inhibits the growth of glioma stem and non-stem cultures, including temozolomide-resistant cell lines, in vitro and in vivo. Cancer Res. 75, 2562 (2015). abstract.

20. Shih, K. C. et al. Phase I/II study of VAL-083 in patients with recurrent glioblastoma. J. Clin. Oncol. 34, 2063 (2016). abstract.

21. ClinicalTrials.govNCT01478178. Safety study of VAL-083 in patients with recurrent malignant glioma. https:/clinicaltrials.gov/ct2/show/NCT01478178. (2011-2016).

22. Institoris, E, Szikla, K, Otvos, L \& Gal, F. Absence of cross-resistance between two alkylating agents: BCNU vs bifunctional galactitol. Cancer Chemother Pharmacol. 24, 311-313 (1989).

23. Kuo, L. J. \& Yang, L. X. Gamma-H2AX - a novel biomarker for DNA doublestrand breaks. In Vivo 22, 305-309 (2008).

24. Podhorecka, M., Skladanowski, A. \& Bozko, P. H2AX phosphonylation: its role in dna damage response and cancer therapy. J. Nucleic Acids 2010, Article ID 920161 (2010) (PMID: 20811597).

25. Heichman, K. A. \& Roberts, J. M. Rules to replicate by. Cell 79, 557-562 (1994).

26. King, R. W., Jackson, P. K. \& Kirschner, M. W. Mitosis in transition. Cell 79 563-571 (1994)

27. Sherr, C. J. G1 phase progression: cycling on cue. Cell 79, 551-555 (1994).

28. Yam, C. H., Fung, T. K. \& Poon, R. Y. Cyclin A in cell cycle control and cancer Cell. Mol. Life Sci. 59, 1317-1326 (2002).

29. Chapman, J. R., Taylor, M. R. \& Boulton, S. J. Playing the end game: DNA double-strand break repair pathway choice. Mol. Cell 47, 497-510 (2012).

30. Sartori, A. A. et al. Human CtIP promotes DNA end resection. Nature $\mathbf{4 5 0}$ 509-514 (2007)

31. You, Z. et al. CtIP links DNA double-strand break sensing to resection. Mol. Cell 36, 954-969 (2009)

32. Daugaard, $M$. et al. LEDGF ( $p 75$ ) promotes DNA-end resection and homologous recombination. Nat. Struct. Mol. Biol. 19, 803-810 (2012).

33. Baude, A. et al. Hepatoma-derived growth factor-related protein 2 promotes DNA repair by homologous recombination. Nucleic Acids Res. 44, 2214-2226 (2016).

34. Liu, S. et al. Distinct roles for DNA-PK, ATM and ATR in RPA phosphorylation and checkpoint activation in response to replication stress. Nucleic Acids Res. 40, 10780-10794 (2012)

35. Feng, L., Fong, K. W., Wang, J., Wang, W. \& Chen, J. RIF1 counteracts BRCA1mediated end resection during DNA repair. J. Biol. Chem. 288, 11135-11143 (2013).

36. Jasin, M. Homologous repair of DNA damage and tumorigenesis: the BRCA connection. Oncogene 21, 8981-8993 (2002).

37. Konstantinopoulos, P. A., Ceccaldi, R., Shapiro, G. I. \& D'Andrea, A. D. Homologous recombination deficiency: exploiting the fundamental vulnerability of ovarian cancer. Cancer Discov. 5, 1137-1154 (2015).

38. Helleday, T., Petermann, E., Lundin, C., Hodgson, B. \& Sharma, R. A. DNA repair pathways as targets for cancer therapy. Nat. Rev. Cancer 8, 193-204 (2008).

39. Institoris, E. In vivo study on alkylation site in DNA by the bifunctional dianhydrogalactitol. Chem. Biol. Interact. 35, 207-216 (1981). 
40. Steino, A. et al. Post-market clinical trial of dianhydrogalactitol in the treatment of relapsed or refractory non-small cell lung cancer. https://ibrary.iaslc.org/ search-speaker?search_speaker=29999 (2015).

41. Fan, C. H. et al. O6-methylguanine DNA methyltransferase as a promising target for the treatment of temozolomide-resistant gliomas. Cell Death Dis. $\mathbf{4}$, e876 (2013).

42. Steino, A. et al. The unique mechanism of action of VAL-083 may provide a new treatment option for some chemo-resistant cancers. Mol. Cancer Ther. 12 B252 (2014). abstract.
43. Ceccaldi, R., Sarangi, P. \& D'Andrea, A. D. The Fanconi anaemia pathway: new players and new functions. Nat. Rev. Mol. Cell Biol. 17, 337-349 (2016).

44. Abraham, R. T. Cell cycle checkpoint signaling through the ATM and ATR kinases. Genes Dev. 15, 2177-2196 (2001).

45. Jackson, S. P. \& Bartek, J. The DNA-damage response in human biology and disease. Nature 461, 1071-1078 (2009).

46. Jackson, S. P. Sensing and repairing DNA double-strand breaks. Carcinogenesis 23, 687-696 (2002) 